\title{
Investigation of Localized Coupled-Cavity Modes in Two-Dimensional Photonic Bandgap Structures
}

\author{
Ekmel Ozbay, Mehmet Bayindir, Irfan Bulu, and Ertugrul Cubukcu
}

\begin{abstract}
We present a detailed study of the localized coupled-cavity modes in 2-D dielectric photonic crystals. The transmission, phase, and delay time characteristics of the various coupled-cavity structures are measured and calculated. We observed the eigenmode splitting, waveguiding through the coupled cavities, splitting of electromagnetic waves in waveguide ports, and switching effect in such structures. The corresponding field patterns and the transmission spectra are obtained from the finite-difference-time-domain (FDTD) simulations. We also develop a theory based on the classical wave analog of the tight-binding (TB) approximation in solid state physics. Experimental results are in good agreement with the FDTD simulations and predictions of the TB approximation.
\end{abstract}

Index Terms-FDTD, localization, photonic bandgap (PBG), splitters, switches, tight-binding approximation, waveguides.

\section{INTRODUCTION}

$\mathbf{P}$ HOTONIC crystals, also known as photonic bandgap (PBG) materials, are artificial dielectric or metallic structures in which the refractive index modulation gives rise to stop bands for electromagnetic waves (EM) within a certain frequency range in all directions [1], [2]. In the last several years, photonic crystals have inspired great interest because of their novel scientific and engineering applications such as localization of light wave [3], the inhibition of spontaneous emission [4], [5], lasers [6]-[8], waveguides [9]-[13], splitters [14]-[16], fibers [17], antennas [18], [19], optical circuits [20], [21], and ultrafast optical switches [22].

By removing from or adding to materials a perfect photonic crystal, it is possible to create localized EM modes inside the PBG which are reminiscent of the acceptor and donor impurity states in a semiconductor [23], [24]. Therefore, photons with certain wavelengths can locally be trapped inside the defect volume. This important property can be used in various photonic applications. In fact, most of the aforementioned applications are based on cavity structures built around photonic crystals.

PBG structures also provide a promising tool to control of the flow EM waves in the integrated optical devices. Therefore, there is a growing interest in developing photonic crystal-based waveguide components which can guide and bend EM waves either along a line defect (a row of missing rods) [10], [13] or through coupled cavities [12]. In the former case, while the EM waves are confined in one direction which is perpendicular to

Manuscript received September 25, 2001; revised February 19, 2002. This work was supported by NATO under Grant SfP971970, by the National Science Foundation under Grant INT-9820646, by the Turkish Department of Defense under Grant KOBRA-001, and by Thales JP8.04.

The authors are with the the Department of Physics, Bilkent University, Bilkent, 06533 Ankara, Turkey (e-mail: bayindir@fen.bilkent.edu.tr).

Publisher Item Identifier S 0018-9197(02)05701-9.

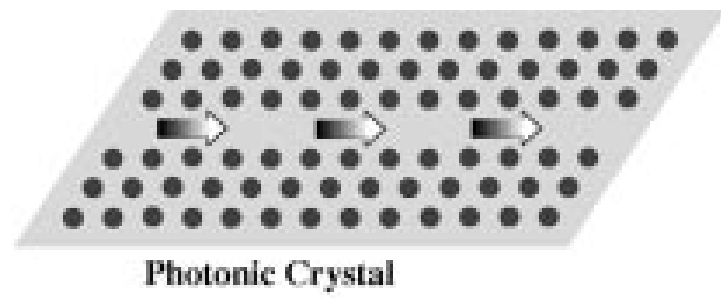

(a)

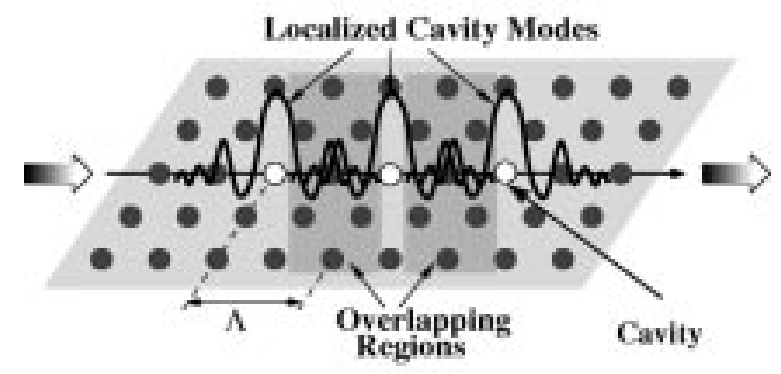

(b)

Fig. 1. Schematic drawing of two different waveguiding mechanism in photonic crystals. (a) Planar waveguides (PW) consist of two parallel photonic crystal mirrors with a suitable separation between them. Photons confined between these two photonic crystal walls, and can propagate through the opening which can be considered a continuous defect. (b) CCWs are formed by removing an array of rods (white circles) from a 2-D photonic crystal (black circles). Tightly confined cavity mode interacts weakly with the neighboring cavity modes, and therefore the electromagnetic waves can propagate through coupled cavities.

the axis of missing rods, and photons can propagate in other directions parallel to the axis of the missing rods [Fig. 1(a)]. On the other hand, in the latter case, which we called coupled-cavity waveguides (CCW) [25], the EM waves were tightly confined at each defect site, and photons can propagate by hopping, due to interactions between the neighboring evanescent cavity modes [Fig. 1(b)].

An analogy between Schrodinger's equation and Maxwell's equations allows us to use many important tools which were originally developed for electronic systems. As an example, it is well known that the TB method has proven to be very useful in studying the electronic properties of solids [26]. Recently, the classical wave analog of the TB picture [27], [28] has successfully been applied to photonic structures [29]-[33], [52], [25]. Sterke investigated the properties of the 1-D optical superlattices within the TB approximation [29]. Lidorikis et al. obtained matrix elements of the TB Hamiltonian for 2-D photonic crystals, with and without defects, and tested the TB model by comparing it to the corresponding ab initio results [31]. Observation of the normal mode splitting in quartz polystyrene was well explained by the TB photon approach [32]. 
By using direct implications of the TB picture, a novel propagation mechanism for photons along localized coupled cavity modes in photonic crystals was theoretically proposed [30], [33], [34] and experimentally demonstrated [25]. In these structures, photons can hop from one tightly confined mode to the neighboring one due to weak interactions between them [see Fig. 1(b)]. Stefanou and Modinos obtained the cosine-like dispersion relation for their coupled-defect waveguide and waveguide bends with and without disorder [30]. Later, Yariv et al. reformulated the same phenomenon in a simple way, and more importantly, proposed various applications based on coupled-cavity structures [33], [34]. Then, we investigated mode-splitting phenomena within the TB picture [25]. Guiding and bending of EM waves [12], heavy photons [35], and the EM-beam splitting and switching effects [36] were experimentally demonstrated in 3-D photonic crystals at microwave frequencies. In addition, we observed the strong enhancement of spontaneous emission throughout the cavity band of 1-D coupled optical microcavity structures [37], [38]. Very recently, Lan et al. numerically proposed a switching mechanism by changing the positions of the sharp edges of the coupled-cavity band of 1-D PBG structures [39] and delay lines for ultrashort optical pulses [40], and Olivier et al. reported 2-D CCWs at optical wavelengths [41]. The coupled-mode theory was applied to the coupled-cavity structure by Reynolds and his co-workers [42].

Since most of the photonic crystal-based applications have been demonstrated in 2-D photonic structures, especially at optical wavelengths, it is important to study 2-D CCWs in details. In the present paper, we will present a comprehensive experimental and theoretical study on 2-D coupled-cavity structures. The paper is organized as follows: In Section II, we first analyze the localized cavity modes within the classical wave analog of the TB approximation in solid state physics. Observation of the eigenmode splitting is described in Section III, and the measured results are compared with the TB predictions. In Section IV, we experimentally demonstrate guiding and bending of EM waves through the CCWs, and numerically obtain the corresponding field patterns. The coupled-cavity based splitting and switching structures are investigated in Section V.

\section{Localized CCW Modes In Photonic CRystals: TB APPROXIMATION}

In this section, we first investigate splitting of eigenmodes of two and three coupled cavities by using the TB approach [25]. Then, we derive simple expressions for dispersion relation, group velocity, and photon lifetime [30], [33], [34], [25]. Now, consider a strongly localized mode $\mathbf{E}_{\Omega}(\mathbf{r})$ corresponding to a single cavity that satisfies simplified version of the Maxwell equations

$$
\nabla \times\left[\nabla \times \mathbf{E}_{\Omega}(\mathbf{r})\right]=\epsilon_{0}(\mathbf{r})(\Omega / c)^{2} \mathbf{E}_{\Omega}(\mathbf{r}),
$$

where $\epsilon_{0}(r)$ is the dielectric constant of the single cavity and $\Omega$ is the frequency corresponding cavity mode. Here, we assumed that $\mathbf{E}_{\Omega}(\mathbf{r})$ is real, nondegenerate and orthonormal, i.e., $\int \mathrm{d} \mathbf{r} \epsilon_{0}(\mathbf{r}) \mathbf{E}_{\Omega}(\mathbf{r}) \cdot \mathbf{E}_{\Omega}(\mathbf{r})=1$.

When two localized cavity modes are brought in contact, the corresponding eigenmode can be obtained from the superposition of the individual evanescent cavity modes $\mathbf{E}_{\omega}(\mathbf{r})=A \mathbf{E}_{\Omega}(\mathbf{r})+B \mathbf{E}_{\Omega}(\mathbf{r}-\Lambda \hat{x})$. The eigenmode $\mathbf{E}_{\omega}(\mathbf{r})$ also satisfies (1) where $\epsilon_{0}(\mathbf{r})$ is replaced with the dielectric constant of the coupled system $\epsilon(\mathbf{r})=\epsilon(\mathbf{r}-\Lambda \hat{x})$, and $\Omega$ is replaced with eigenfrequency $\omega$ of the coupled cavity mode. Inserting $\mathbf{E}_{\omega}(\mathbf{r})$ into (1), and multiplying both sides from the left first by $\mathbf{E}_{\Omega}(\mathbf{r})$ and then by $\mathbf{E}_{\Omega}(\mathbf{r}-\Lambda \hat{x})$ and spatially integrating the resulting equations, we obtain the following eigenmodes and eigenfrequencies:

$$
\begin{aligned}
\mathbf{E}_{\omega_{1,2}}(\mathbf{r}) & =\frac{\mathbf{E}_{\Omega}(\mathbf{r}) \pm \mathbf{E}_{\Omega}(\mathbf{r}-\Lambda \hat{x})}{\sqrt{2}} \\
\omega_{1,2} & =\Omega \sqrt{\frac{1 \pm \beta_{1}}{1 \pm \alpha_{1}}}
\end{aligned}
$$

where $\alpha_{1}$ and $\beta_{1}$ are the first order coupling parameters which are given by $\alpha_{1}=\int \operatorname{dr} \epsilon(\mathbf{r}) \mathbf{E}_{\Omega}(\mathbf{r}) \cdot \mathbf{E}_{\Omega}(\mathbf{r}-\Lambda \hat{x})$, and $\beta_{1}=$ $\int \mathrm{d} \mathbf{r} \epsilon_{0}(\mathbf{r}-\Lambda \hat{x}) \mathbf{E}_{\Omega}(\mathbf{r}) \cdot \mathbf{E}_{\Omega}(\mathbf{r}-\Lambda \hat{x})$.

Similarly, the eigenmodes of three coupled cavities can be obtained in a similar way

$$
\begin{aligned}
\mathbf{E}_{\Gamma_{1,3}}(\mathbf{r}) & =\frac{\mathbf{E}_{\Omega}(\mathbf{r}) \pm \sqrt{2} \mathbf{E}_{\Omega}(\mathbf{r}-\Lambda \hat{x})+\mathbf{E}_{\Omega}(\mathbf{r}-2 \Lambda \hat{x})}{2}, \\
\mathbf{E}_{\Gamma_{2}}(\mathbf{r}) & =\frac{\mathbf{E}_{\Omega}(\mathbf{r})-\mathbf{E}_{\Omega}(\mathbf{r}-2 \Lambda \hat{x})}{\sqrt{2}} .
\end{aligned}
$$

The corresponding eigenvalues are given by

$$
\begin{aligned}
\Gamma_{2} & =\Omega \sqrt{\frac{1-\beta_{2}}{1-\alpha_{2}}} \\
\Gamma_{1,3} & =\Omega \sqrt{\frac{1 \pm \sqrt{2} \beta_{1}+\beta_{2} / 2}{1 \pm \sqrt{2} \alpha_{1}+\alpha_{2} / 2}}
\end{aligned}
$$

where $\alpha_{2}$ and $\beta_{2}$ are the second nearest neighbor coupling terms which are given by $\alpha_{2}=\int \mathrm{d} \mathbf{r} \epsilon(\mathbf{r}) \mathbf{E}_{\Omega}(\mathbf{r}) \cdot \mathbf{E}_{\Omega}(\mathbf{r}-2 \Lambda \hat{x})$, and $\beta_{2}=\int \mathrm{d} \mathbf{r} \epsilon_{0}(\mathbf{r}-2 \Lambda \hat{x}) \mathbf{E}_{\Omega}(\mathbf{r}) \cdot \mathbf{E}_{\Omega}(\mathbf{r}-2 \Lambda \hat{x})$.

When we consider an array of cavities in which each cavity interacts weakly with neighboring cavities, a defect band (waveguiding band) is formed. The eigenmode of this waveguiding band can be written as a superposition of the individual cavity modes which is analogous to the linear combination of atomic orbitals in solid-state physics

$$
\mathbf{E}(\mathbf{r})=E_{0} \sum_{n} e^{-i n k \Lambda} \mathbf{E}_{\Omega}(\mathbf{r}-n \Lambda \hat{x})
$$

where the summation over $n$ includes all the cavities. The dispersion relation for this structure can be obtained from (1) and (7), keeping only the first two coupling terms

$$
\omega(k)=\Omega\left(\frac{\beta_{1} \cos (k \Lambda)+2 \beta_{2} \cos (2 k \Lambda)+1 / 2}{\alpha_{1} \cos (k \Lambda)+2 \alpha_{2} \cos (2 k \Lambda)+1 / 2}\right)^{1 / 2} .
$$

In certain cases, we can safely ignore the second nearest neighbor terms, and this approximation leads to a simpler expression for the dispersion relation

$$
\omega(k)=\Omega[1+\kappa \cos (k \Lambda)] .
$$

Here, $\kappa=\beta_{1}-\alpha_{1}$ is a TB parameter which can be obtained from the splitting of the eigenmodes of two coupled cavities. 
After obtaining $\Omega, \omega_{1}$, and $\omega_{2}$ from measurements or simulations, one can determine $\beta_{1}$ and $\alpha_{1}$ values by using (3). The bandwidth of the waveguiding band is proportional to the coupling constant and single cavity frequency, which is given by

$$
\Delta \omega=2 \kappa \Omega_{0} .
$$

The group velocity of photons along the coupled cavities can be determined from the dispersion relation

$$
v_{g}(k)=\nabla_{k} \omega_{k}=-\kappa \Lambda \Omega \sin (k \Lambda) .
$$

The net phase difference $\Delta \varphi$ is related to the wave vector $k$ of the crystal

$$
k L-k_{0} L=\Delta \varphi
$$

where $L$ is the total crystal thickness, $k_{0}=2 \pi \omega / c$, and $c$ is the speed of light in vacuum. Combining (9) and (12) along with the definition $\tau_{p}=\partial(\Delta \varphi) / \partial \omega$, we obtained a formula for delay time (photon lifetime) as a function of frequency

$$
\tau_{p}(\omega)=\frac{L / \Lambda}{\Omega \sqrt{\kappa^{2}-(\omega / \Omega-1)^{2}}}-2 \pi L / c .
$$

This formula indicates that the delay time increases rapidly as we approach the waveguiding bandedges.

It is important to note that all physical quantities including dispersion relation and group velocity depend on only a single TB parameter $\kappa$, and this parameter can be controlled by changing the properties of cavities and the intercavity distance. The coupled-cavity structures can efficiently be used in certain applications such as dispersion compensators and photonic switches. Moreover, the spontaneous emission rate and the efficiency of nonlinear processes can be enhanced in coupled-cavity systems due to low group velocity and a large photon lifetime.

\section{EIGENMODE SPLITTING: EXPERIMENT VERSUS THEORY}

We first constructed 2-D triangular photonic crystals which consist of dielectric cylindrical alumina rods having radius of $1.55 \mathrm{~mm}$ and a refractive index of 3.1 at microwave frequencies. The lattice constant and the corresponding filling fraction are $a=1.3 \mathrm{~cm}$ and $\eta \sim 0.05$, respectively. The length of the rods is $15 \mathrm{~cm}$. The experimental setup consisted of a HP 8510C network analyzer and microwave horn antennas to measure the transmission-amplitude and the transmission-phase properties. The transverse magnetic (TM) polarization, the incident electric field was parallel to the rods, is considered in all measurements. It is well known that the other polarization, transverse electric (TE), does not produce any PBGs in this frequency range [1]. The transmission spectra and the field patterns are obtained by using FullWave which is a commercially available finite-difference-time-domain (FDTD) code. In our experiments, normalization of the transmission and the phase data is done as follows. First, we measured the transmission and phase spectra in the free space (i.e., without photonic crystal). This data was used as the calibration data for the network analyzer. Then, we inserted the crystal between the horn antennas, and we performed the transmission and phase measurements by keeping the distance between the transmitter and receiver antennas fixed. In FDTD sim-

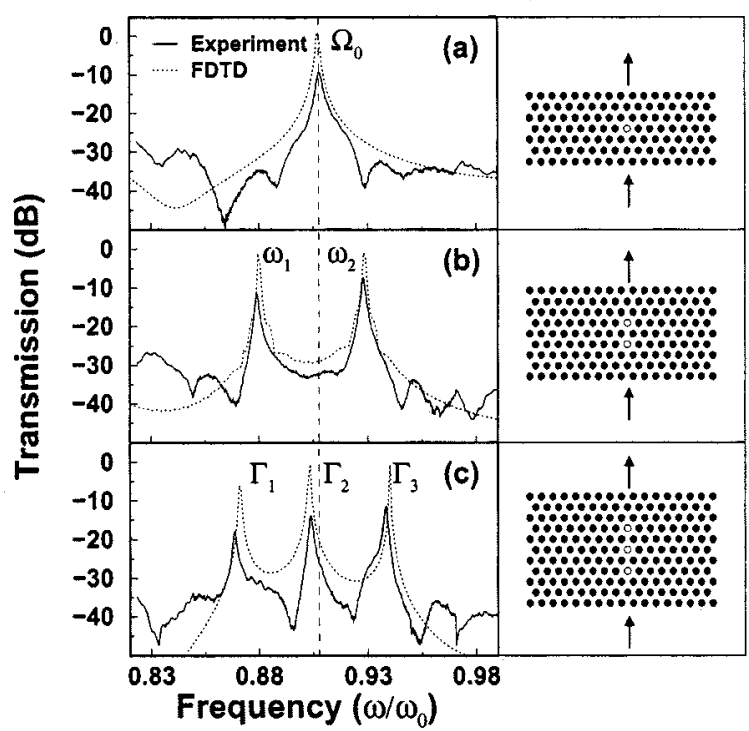

Fig. 2. Left panels: Measured (solid lines) and calculated (dotted lines) transmission spectra by using FDTD method for: (a) a single cavity; (b) two coupled-cavity; and (c) three coupled-cavity structures. Due to coupling between the strongly localized cavity modes, single cavity mode splits into two or three distinct modes depending on the number of coupled cavities. There is good agreement between the measured and calculated transmission spectra. Right panels: Schematics of the corresponding coupled-cavity structures where (o) symbols denote the removed rods.

ulations, we normalized the transmission spectra with respect to the source spectra.

The crystal exhibits a PBG extending from $0.73 \omega_{0}$ to $1.14 \omega_{0}$ where $\omega_{0}=c / 2 a=11.54 \mathrm{GHz}$. Fig. 2 (left panel) shows the measured and calculated transmission characteristics of various coupled-cavity structures. Corresponding schematic drawings of these structures are displayed in the right panel of Fig. 2. When we remove a single rod from an otherwise perfect crystal, a highly localized cavity mode appears at frequency $\Omega_{0}=0.908 \omega_{0}$ having a quality factor of 900 [Fig. 2(a)]. Next, we measure and calculate the transmission through a crystal which contains two coupled cavities with an intercavity distance $\Lambda=\sqrt{3} a$. As shown in Fig. 2(b), we observe that the single cavity mode is split into two resonance modes at $\omega_{1}=0.879 \omega_{0}$ and $\omega_{2}=0.928 \omega_{0}$ due to interaction between two adjacent localized cavity modes.

Localized defect modes in 2-D photonic crystals are widely studied in [43]-[45], and splitting of an eigenmode is already reported in various photonic structures. This phenomenon is observed in (3-D diamond wire mesh photonic crystals [46], coupled dielectric spheres [47], [48], coupled pairs of micrometer-sized semiconductor cavities [46], two polymer bispheres in contact [32], and coupled defects in 3-D dielectric photonic crystals [25].

In the case of three coupled cavities, the eigenmode splits into three resonance frequencies. As shown in Fig. 2(c), there is a good agreement between measured and simulated results. We also compare the TB predictions of the resonant frequencies $\Gamma_{1,2,3}$ with the measured and FDTD simulation results (see Table I). In order to calculate these frequencies, we first determine the first order TB parameters $\alpha_{1}$ and $\beta_{1}$ by inserting the measured values of $\omega_{1}$ and $\omega_{2}$ into (3). Then, 
TABLE I

COMPARISON OF THE RESONANT FREQUENCIES OF THREE COUPLED CAVITIES OBTAINED By MEASUREMENTS, THE FDTD SiMULATIONS, AND THE TB ApProximation. THE MEASURED Results aRE IN EXCELLENT AgREEMENT With THE FDTD SimULATION RESUlTS, AND THE PREDICTION OF THE TB APPROXIMATION

\begin{tabular}{c||c|c|c|c}
\hline & & & & \\
& Measurement & FDTD & TB & TB $^{\dagger}$ \\
\hline \hline$\Gamma_{1}$ & 0.869 & 0.871 & 0.863 & 0.866 \\
$\Gamma_{2}$ & 0.904 & 0.903 & 0.908 & 0.904 \\
$\Gamma_{3}$ & 0.938 & 0.940 & 0.935 & 0.937 \\
\hline
\end{tabular}

$\dagger$ Second nearest neighbors coupling was included.

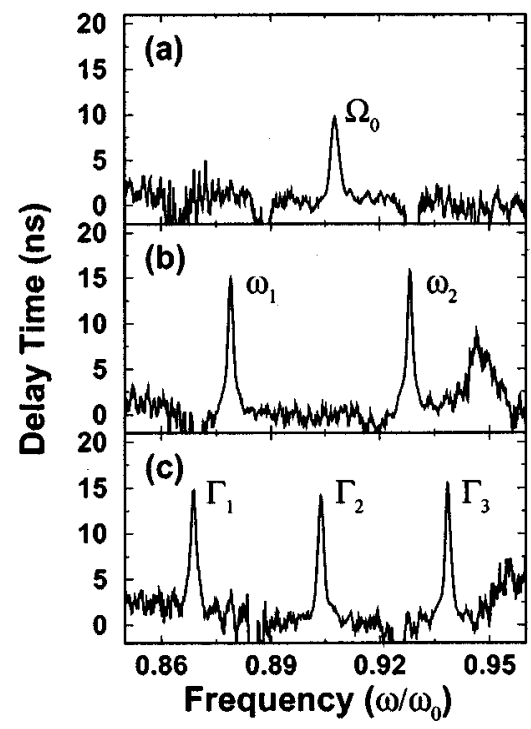

Fig. 3. Measured delay time characteristics of the corresponding coupled-cavity structures, namely: (a) single cavity; (b) two coupled-cavity; and (c) three coupled-cavity. $\tau_{p}$ exhibit peaks at the resonance frequencies which implies that photons spend more time inside the cavities for these frequencies.

$\Gamma_{1,2,3}$ are obtained by using these parameters in (6) where the second nearest neighbor coupling terms are ignored. As shown in Table I, the predictions of the TB approximation agree well with the measured and the FDTD results. When the second nearest neighbor coupling terms $\alpha_{2}$ and $\beta_{2}$ [51] are included into (6), better agreement is obtained, especially for $\Gamma_{2}$.

We also measured the delay time corresponding to these coupled-cavity structures. In experiments, the delay time $\tau_{p}$ is obtained from the phase measurements via $\tau_{p}=\partial(\Delta \varphi) / \partial \omega$. Here, $\Delta \varphi$ is the net phase difference between the phase of the EM wave propagating through the photonic crystal and the phase of the EM wave propagating in free space. Fig. 3 displays the measured delay time characteristics as a function of frequency. Drastic enhancement of $\tau_{p}$ at the resonance frequencies implies that the EM waves are confined inside the cavities, and therefore propagate more slowly compared to the free space EM waves.

\section{Coupled-CAVity WaVEGuides}

The efficient guiding and bending of light by integrated photonic devices are important to designing optical circuits for tech-

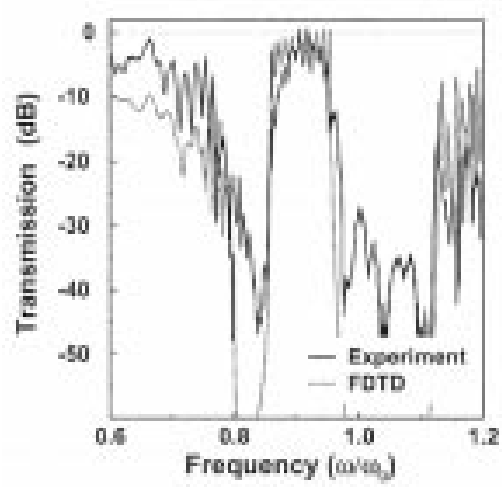

(a)

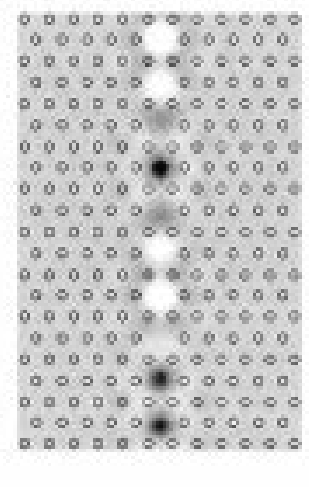

(b)
Fig. 4. (a) Measured (solid line) and calculated (dotted line) transmission spectra of the straight $\mathrm{CCW}$ which is generated by removing 10 adjacent rods from the crystal. A cavity band is formed extending from $0.857 \omega_{0}$ to $0.956 \omega_{0}$. Nearly $100 \%$ transmission is achieved for certain frequencies. (b) Calculated field distribution of the $\mathrm{CCW}$ for a frequency within the cavity band, namely, $\omega=0.920 \omega_{0}$. The guided mode travels along the cavities due to coupling between the localized cavity modes. The electric field $\mathbf{E}$ is polarized along the axis of missing rod.

nological and optical computing applications. Also, the problem of guiding light around sharp corners must be addressed. Conventional dielectric or metallic waveguides have large scattering losses when sharp bends are introduced. In recent years, it has been demonstrated that photonic crystal based waveguides can efficiently guide and bend EM waves [9], [12].

In this section, we investigate the transmission and phase properties of straight and zig-zag CCWs. We first measure and calculate the transmission spectra of a straight $\mathrm{CCW}$ that is formed by removing an array of rods, 10 consecutive defects, along a straight line. The corresponding structure is displayed in Fig. 4(b) (o symbols). A waveguiding band, or defect band, is formed due to coupling between the evanescent defect modes. Recently, Bayer et al. observed formation of a photonic band due to coupling between the optical molecules [52], [53].

As shown in Fig. 4(a), the defect band extends from $0.857 \omega_{0}$ to $0.956 \omega_{0}$. The number of peaks in the transmission spectrum is equal to the number of cavities in the structure as expected. It is also observed that complete transmission is achieved for certain frequencies within the defect band. Since each mode is strongly localized around the removed rod, and the guided mode is composed of a linear combination of these individual defect modes, we expect that the radiation loss mechanism is absent in these structures. We also calculate the corresponding field distribution of the guided mode for $\omega=0.920 \omega_{0}$. Fig. 4(b) clearly shows that the guided mode is completely confined along with the coupled-cavity array, and propagates along with the cavity sites.

Then, we measure and calculate the transmission characteristics of a zig-zag waveguide which consists of 16 coupled cavities. The distance between cavities are the same, even if the propagation direction is changed arbitrarily. Fig. 5(a) displays corresponding transmission spectra as a function of normalized frequency. A defect band is formed between $0.857 \omega_{0}$ and $0.949 \omega_{0}$. We also achieve complete transmission for certain frequencies within the defect band. Although the zig-zag CCWs have many sharp corners, these waveguides exhibit properties similar to those we obtained for the straight CCWs. 


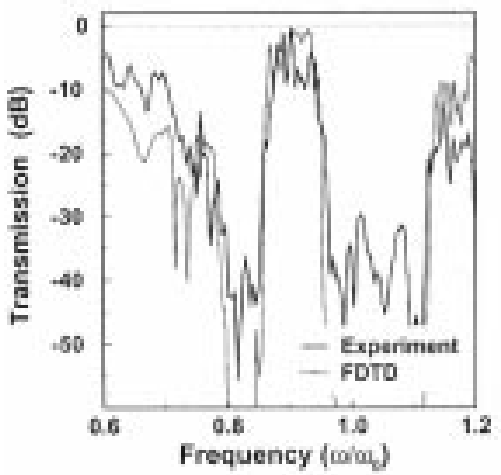

(a)

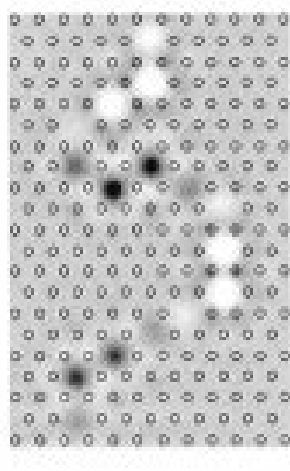

(b)
Fig. 5. (a) Measured (solid line) and calculated (dotted line) transmission spectra of the zig-zag CCW which contains 16 cavities. (b) Calculated field distribution of the zig-zag CCW for a frequency within the cavity band, namely, $\omega=0.936 \omega_{0}$

Based on our observations, the crystal symmetry has a crucial role in 2-D photonic crystals in achieving complete transmission of EM waves through CCWs [33], [34]. For instance, even if the triangular symmetry leads to nearly full transmissions, one can obtain only $10 \%$ transmission for the square photonic crystals [54]. We think that this observation is related to the localization properties of the cavity mode, and the nature of the interaction between the cavity modes.

It is important to note that the TB parameter $\kappa$ can also be determined from the bandwidth of the defect band by using $|\kappa|=\Delta \omega / 2 \Omega_{0}=0.054$. This result is close to the previously obtained value in Section III. In addition, the bandwidth of a $\mathrm{CCW}$ band can be adjusted by changing localization properties of the cavities, or the coupling strength (overlap integral) between the localized cavity modes. For instance, decreasing the intercavity distance leads to a wider bandwidth.

Previously, Scalora et al. have proposed a switching mechanism which can be achieved by dynamical shifting of the PBG edges via nonlinear processes [51]. As shown from Fig. 4, the defect band has sharp band edges compared to the PBG edges, and, therefore, this property can be used to construct photonic switches by changing the position of the defect band [12], [39].

We also determine dispersion relation, group velocity, and delay time corresponding to the straight CCWs. The dispersion relation $\omega(k)$ is obtained from the transmission-phase information [50], [25]. By measuring the net phase difference $\Delta \varphi$, as a function of frequency $\omega$, the wave vector $k$ of the crystal can be determined directly by using (12). Fig. 6(a) exhibits a comparison of measured and calculated dispersion relations as a function of wave vector $k$. The theoretical curve is obtained by using (9) with the experimentally determined TB parameter $\kappa=-0.0525$. We also plot the group velocity of the propagating mode by using (11). The experimental curve is determined from the measured dispersion relation. As shown in Fig. 6(b), the group velocity vanishes at the defect band edges, and the maximum value of the group velocity is one order of magnitude smaller than the speed of light.

The measured and calculated delay time characteristics are displayed in Fig. 6(c). The delay time increases drastically at the CCW band edges. This result agrees well with our TB analysis
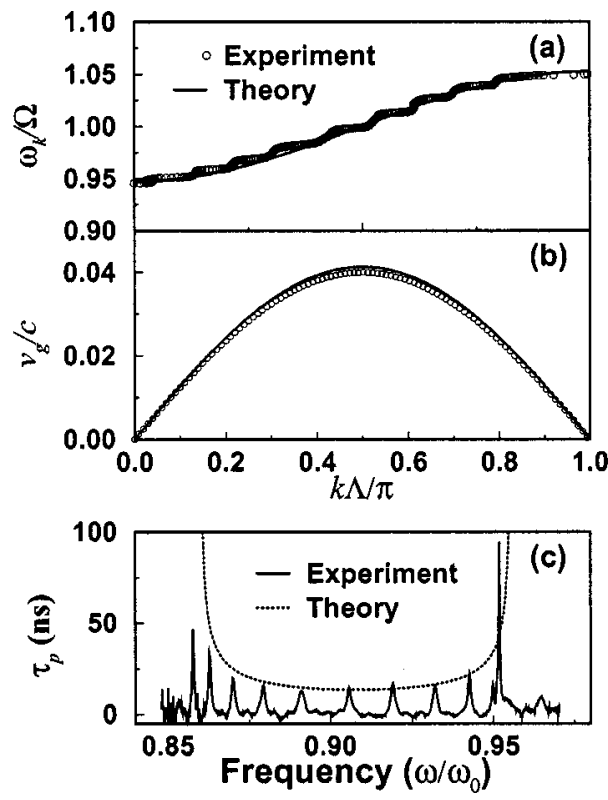

Fig. 6. Comparison of the measured and calculated: (a) dispersion relation; (b) group velocity; and (c) delay time corresponding to the straight CCW. Equations (9), (11), and (13) are used to determine $\omega(k), v_{g}$, and $\tau_{p}$ with $\kappa=-0.0525$, respectively. There is good agreement between the experimental results and the predictions of the tight-binding approximation.

(dotted line). The number of observed peaks in the delay time spectrum is equal to the number of cavities used in the structure. Physically, the heavy photon concept in the PBG structures is reminiscent of the heavy electron in semiconductors having energies near the band edges. The corresponding eigenfunctions are standing waves rather than the propagating waves, and therefore the effective mass of electrons becomes large [28].

\section{SPlitTERS AND SWITCHES}

Splitters and switches are important components for designing photonic circuits. In recent years, many authors have reported splitters and switches which are built around PBG structures [14]-[16], [36]. In this section, we demonstrate the splitting of propagating waves into two output waveguide ports. We also report a switching mechanism based on coupled-cavity structures.

In order to demonstrate the splitting of EM power, we construct a Y-shaped splitter which consists of one input CCW and two output CCWs. The input and output waveguide ports contain five and six coupled cavities, respectively. As shown in Fig. 7(a), the propagating mode inside the input CCW splits equally into two output $\mathrm{CCW}$ ports for all frequencies within the defect band. We also compute the electric field distribution inside the input and output $\mathrm{CCW}$ channels for a frequency $\omega=0.916 \omega_{0}$.

In the present structure, the splitter structure can also be used as a photonic switch. To demonstrate the switching effect, we place a single rod to the left side of the junction of the Y-splitter. This breaks the symmetry of the structure, and, therefore, one can regulate the amount of power flow into the output ports of CCWs. As shown in Fig. 8, the power at each output waveguide port is drastically changed. While most of the input power for 


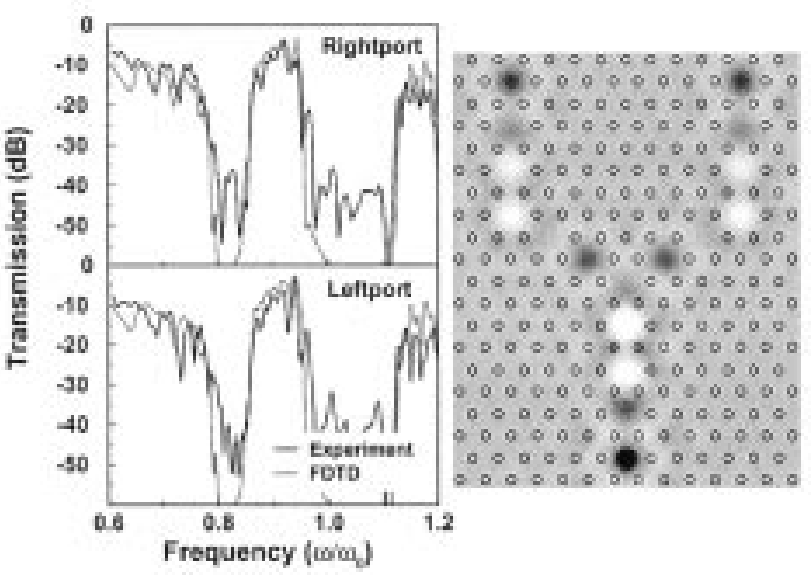

(a)

(b)

Fig. 7. (a) Measured (solid line) and calculated (dotted line) of transmission spectra of a Y-shaped coupled-cavity based splitter. The electromagnetic power in the input port splits equally into the two output waveguide ports for frequencies throughout the cavity band. (b) Calculated power distribution inside the input and the output waveguide channels of the splitter for frequency $\omega=0.916 \omega_{0}$.

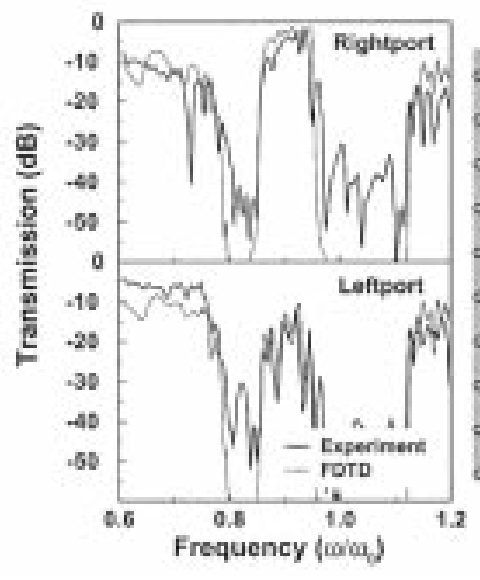

(a)

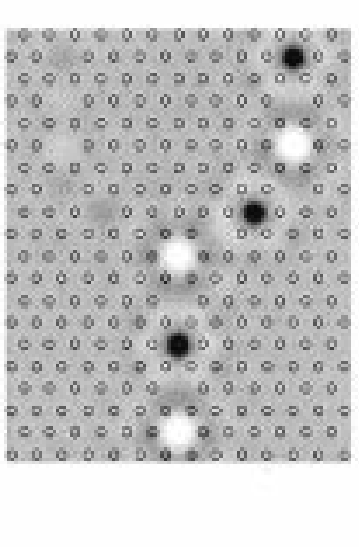

(b)
Fig. 8. (a) Measured (solid line) and calculated (dotted line) transmission characteristics of a coupled-cavity switching structure. (b) Corresponding field pattern clearly indicates that most of the power is coupled to the right port.

frequencies throughout the defect band is coupled to the right port, only a few percent of the input power is coupled to the left port. The simulated electric field distribution is also displayed in Fig. 8(b).

\section{CONCLUSION}

In summary, a comprehensive study of the coupled-cavity structures in 2-D triangular dielectric photonic crystals has been presented. The phenomenon of eigenmode splitting is observed when the isolated localized coupled-cavity modes are brought into contact. A new type of waveguiding mechanism, the propagation of photons by hopping, is demonstrated in 2-D photonic crystals. Nearly full transmission of electromagnetic waves is achieved, even if the CCWs contain very sharp bends and the propagation direction is arbitrarily changed. $\mathrm{CCW}$ structures are also used to design power splitters and photonic switches. These results are important for designing efficient waveguide components in photonic crystal based optical circuits. The simulated mode patterns and the transmission characteristics by using an FDTD code agree well with our tight-binding model's predictions. This excellent agreement is an indication of the usefulness of the tight-binding formalism to investigate the interaction between evanescent electromagnetic modes in photonic structures.

\section{REFERENCES}

[1] J. D. Joannopoulos, R. D. Meade, and J. N. Winn, Photonic Crystals: Molding the Flow of Light. Princeton, NJ: Princeton University Press, 1995.

[2] C. M. Soukoulis, Ed., Photonic Crystals and Light Localization in the 21st Century. Norwell, MA: Kluwer, 2001.

[3] S. John, "Strong localization of photons in certain disordered dielectric superlattices," Phys. Rev. Lett., vol. 58, pp. 2486-2489, 1987.

[4] E. Yablonovitch, "Inhibited spontaneous emission in solid-state physics and electronics," Phys. Rev. Lett., vol. 58, pp. 2059-2062, 1987.

[5] M. Boroditsky, R. Vrijen, T. F. Krauss, R. Coccioli, R. Bhat, and E. Yablonovitch, "Spontaneous emission extraction and Purcell enhancement from thin-film 2-D photonic crystals," J. Lightwave Technol., vol. 17, pp. 2096-2112, 1999.

[6] O. Painter, R. K. Lee, A. Scherer, A. Yariv, J. D. O’Brien, P. D. Dapkus, and I. Kim, "Two-dimensional photonic band-gap defect mode laser," Science, vol. 284, pp. 1819-1821, 1999.

[7] A. Mekis, M. Meier, A. Dodabalapur, R. E. Slusher, and J. D. Joannopoulos, "Lasing mechanism in two-dimensional photonic crystal lasers," Appl. Phys. A: Materials Science \& Processing, vol. 69, pp. 111-114, 1999.

[8] S. Noda, M. Yokoyama, M. Imada, A. Chutinan, and M. Mochizuki, "Polarization mode control of two-dimensional photonic crystal laser by unit cell structure design," Science, vol. 293, pp. 1123-1125, 2001.

[9] A. Mekis, J. C. Chen, I. Kurland, S. Fan, P. R. Villeneuve, and J. D. Joannapoulos, "High transmission through sharp bends in photonic crystal waveguides," Phys. Rev. Lett., vol. 77, pp. 3787-3790, 1996.

[10] S.-Y. Lin, E. Chow, V. Hietala, P. R. Villeneuve, and J. D. Joannopoulos, "Experimental demonstration of guiding and bending of electromagnetic waves in a photonic crystal," Science, vol. 282, pp. 274-276, 1998.

[11] B. Temelkuran and E. Ozbay, "Experimental demonstration of photonic crystal based waveguides,” Appl. Phys. Lett., vol. 74, pp. 486-488, 1999.

[12] M. Bayindir, B. Temelkuran, and E. Ozbay, "Propagation of photons by hopping: A waveguiding mechanism through localized coupled-cavities in three-dimensional photonic crystals," Phys. Rev. B, vol. 61, pp. R11855-R11858, 2000.

[13] M. Loncar, D. Nedeljkovic, T. Doll, J. Vuckovic, A. Scherer, and T. P. Pearsall, "Waveguiding in planar photonic crystals," Appl. Phys. Lett., vol. 77, pp. 1937-1939, 2000.

[14] J. Yonekura, M. Ikeda, and T. Baba, "Analysis of finite 2-d photonic crystals of columns and lightwave devices using the scattering matrix method," J. Lightwave Technol., vol. 17, pp. 1500-1508, 1999.

[15] R. W. Ziolkowski and M. Tanaka, "FDTD analysis of PBG waveguides, power splitters and switches," Opt. Quant. Electron., vol. 31, pp. 843-855, 1999.

[16] T. Sondergaard and K. H. Dridi, "Energy flow in photonic crystal waveguides," Phys. Rev. B, vol. 61, pp. 15 688-15696, 2000.

[17] J. C. Knight, J. Broeng, T. A. Birks, and P. St. J. Russell, "Photonic band gap guidance in optical fibers," Science, vol. 282, pp. 1476-1479, 1998.

[18] E. R. Brown, C. D. Parker, and E. Yablonovitch, "Radiation properties of a planar antenna on a photonic-crystal substrate," J. Opt. Soc. Amer. $B$, vol. 10, pp. 404-407, 1993.

[19] B. Temelkuran, M. Bayindir, E. Ozbay, R. Biswas, M. M. Sigalas, G. Tuttle, and K. M. Ho, "Photonic crystal based resonant antenna with a very high directivity,” J. Appl. Phys., vol. 87, pp. 603-605, 2000.

[20] H. Kosaka, T. Kawashima, A. Tomita, M. Notomi, T. Tamamura, T. Sato, and S. Kawakamib, "Photonic crystals for micro lightwave circuits using wavelength-dependent angular beam steering," Appl. Phys. Lett., vol. 74, pp. 1370-1372, 1999.

[21] A. de Lustrac, F. Gadot, S. Cabaret, J.-M. Lourtioz, T. Brillat, A. Priou, and A. E. Akmansoy, "Experimental demonstration of electrically controllable photonic crystals at centimeter wavelengths," Appl. Phys. Lett., vol. 75, pp. 1625-1627, 1999.

[22] P. R. Villeneuve, D. S. Abrams, S. Fan, and J. D. Joannopoulos, "Single-mode waveguide microcavity for fast optical switching," Opt. Lett., vol. 21, pp. 2017-2019, 1996. 
[23] E. Yablonovitch, T. J. Gmitter, R. D. Meade, A. M. Rappe, K. D Brommer, and J. D. Joannopoulos, "Donor and acceptor modes in photonic band structure," Phys. Rev. Lett., vol. 67, pp. 3380-3383, 1991.

[24] E. Ozbay, G. Tuttle, M. M. Sigalas, C. M. Soukoulis, and K. M. Ho, "Defect structures in a layer-by-layer photonic band-gap crystal," Phys. Rev. B, vol. 51, pp. 13961-13965, 1995.

[25] M. Bayindir, B. Temelkuran, and E. Ozbay, "Tight-binding description of the coupled defect modes in three-dimensional photonic crystals," Phys. Rev. Lett., vol. 84, pp. 2140-2143, 2000.

[26] W. A. Harrison, Electronic Structure and the Properties of Solids. San Francisco, CA: Freeman, 1980.

[27] N. W. Ashcroft and N. D. Mermin, Solid State Physics. Philadelphia, PA: Saunders, 1976

[28] C. Kittel, Introduction to Solid State Physics, 7th ed. New York: Wiley, 1996, p. 75.

[29] C. Martijn de Sterke, "Superstructure gratings in the tight-binding approximation," Phys. Rev. E, vol. 57, pp. 3502-3509, 1998.

[30] N. Stefanou and A. Modinos, "Impurity bands in photonic insulators," Phys. Rev. B, vol. 57, pp. 12 127-12133, 1998

[31] E. Lidorikis, M. M. Sigalas, E. N. Economou, and C. M. Soukoulis, "Tight-binding parametrization for photonic band gap materials," Phys. Rev. Lett., vol. 81, pp. 1405-1408, 1998.

[32] T. Mukaiyama, K. Takeda, H. Miyazaki, Y. Jimba, and M. Kuwata-Gonokami, "Tight-binding photonic molecule modes of resonant bispheres," Phys. Rev. Lett., vol. 82, pp. 4623-4626, 1999.

[33] A. Yariv, Y. Xu, R. K. Lee, and A. Scherer, "Coupled-resonator optical waveguide: A proposal and analysis," Opt. Lett., vol. 24, pp. 711-713, 1999.

[34] Y. Xu, R. K. Lee, and A. Yariv, "Propagation and second-harmonic generation of electromagnetic waves in a coupled-resonator optical waveguide," J. Opt. Soc. Amer. B, vol. 17, pp. 387-400, 2000

[35] M. Bayindir and E. Ozbay, "Heavy photons at coupled-cavity waveguide band edges in a three-dimensional photonic crystal," Phys. Rev. B, vol. 62, pp. R2247-R2250, 2000.

[36] M. Bayindir, B. Temelkuran, and E. Ozbay, "Photonic crystal based beam splitters," Appl. Phys. Lett., vol. 77, pp. 3902-3904, 2000.

[37] M. Bayindir, S. Tanriseven, and E. Ozbay, "Propagation of light through localized coupled-cavity modes in one-dimensional photonic band-gap structures," Appl. Phys. A: Mater. Sci. Process, vol. 72, pp. 117-119, 2001.

[38] M. Bayindir, S. Tanriseven, A. Aydinli, and E. Ozbay, "Strong enhancement of spontaneous emission in amorphous-silicon-nitride photonic crystal based coupled-microcavity structures," Appl. Phys. A: Mater. Sci. Process, vol. 73, pp. 125-127, 2001.

[39] S. Lan, S. Nishikawa, and O. Wada, "Leveraging deep photonic band gaps in photonic crystal impurity bands," Appl. Phys. Lett., vol. 78, pp. 2101-2103, 2001.

[40] S. Lan, S. Nishikawa, H. Ishikawa, and O. Wada, "Design of impurity band-based photonic crystal waveguides and delay lines for ultrashort optical pulses," J. Appl. Phys., vol. 90, pp. 4321-4327, 2001.

[41] S. Olivier, C. Smith, M. Rattier, H. Benisty, C. Weisbuch, T. Krauss, R. Houdre, and U. Oesterle, "Miniband transmission in a photonic crystal coupled-resonator optical waveguide," Opt. Lett., vol. 26, pp. 1019-1021, 2001.

[42] A. L. Reynolds, U. Peschel, F. Lederer, P. J. Roberts, T. F. Krauss, and P. J. I. de Maagt, "Coupled defects in photonic crystals," IEEE Trans. Microwave Theory Tech., vol. 49, pp. 1860-1867, 2001.

[43] G. Tayeb and D. Maystre, "Rigorous theoretical study of finite-size two-dimensional photonic crystals doped by microcavities," J. Opt. Soc. Amer. A, vol. 14, pp. 3323-3332, 1997.

[44] V. Kuzmiak and A. A. Maradudin, "Localized defect modes in a two-dimensional triangular photonic crystal," Phys. Rev. B, vol. 57, pp. $15242-15250,1998$

[45] A. M. Zheltikov, S. A. Magnitski, and A. V. Tarasishin, "Two-dimensional photonic crystals with a lattice defect: Spectrum of defect modes, localization of light, and formation of evanescent waves," J. Exp. Theor. Phys., vol. 90, pp. 600-608, 2000.

[46] D. F. Sievenpiper, M. E. Sickmiller, and E. Yablonovitch, "3D wire mesh photonic crystals," Phys. Rev. Lett., vol. 76, pp. 2480-2483, 1996.

[47] M. I. Antonoyiannakis and J. B. Pendry, "Mie resonances and bonding in photonic crystals," Europhys. Lett., vol. 40, pp. 613-618, 1997.

[48] M. I. Antonoyiannakis and J. B. Pendry, "Electromagnetic forces in photonic crystals," Phys. Rev B, vol. 60, pp. 2363-2374, 1999.
[49] M. Bayer, T. Gutbrod, J. P. Reithmaier, A. Forchel, T. L. Reinecke, P. A Knipp, A. A. Dremin, and V. D. Kulakovskii, "Optical modes in photonic molecules," Phys. Rev. Lett., vol. 81, pp. 2582-2585, 1998.

[50] E. Ozbay, E. Michel, G. Tuttle, R. Biswas, K. M. Ho, J. Bostak, and D. M. Bloom, "Terahertz spectroscopy of three-dimensional photonic band gap crystal," Opt. Lett., vol. 19, pp. 1155-1157, 1994.

[51] "These parameters are determined similar to the first order case. Experimental values of $\omega_{1,2}$ are obtained by increasing the intercavity distance to $2 \Lambda, "$.

[52] M. Bayer, T. Gutbrod, A. Forchel, T. L. Reinecke, P. A. Knipp, R. Werner, and J. P. Reithmaier, "Optical demonstration of a crystal band structure formation," Phys. Rev. Lett., vol. 83, pp. 5374-5377, 1999.

[53] G. Guttroff, M. Bayer, J. P. Reithmaier, A. Forchel, P. A. Knipp, and T. L. Reinecke, "Photonic defect states in chains of coupled microresonators," Phys. Rev B, vol. 64, p. 155313, 2001.

[54] M. Bayindir, E. Cubukcu, I. Bulu, T. Tut, E. Ozbay, and C. M. Soukoulis, "Photonic band gaps, defect characteristics, and waveguiding in twodimensional disordered dielectric and metallic photonic crystals," Phys. Rev. B, vol. 64, p. $195113,2001$.

[55] M. Scalora, J. P. Dowling, C. M. Bowden, and M. J. Bloemer, "Optica limiting and switching of ultrashort pulses in nonlinear photonic band gap materials," Phys. Rev. Lett., vol. 73, p. 1368, 1994.

Ekmel Ozbay was born in 1966 in Ankara, Turkey. He received the B.S. degree in electrical engineering from the Middle East Technical University, Ankara, in 1983, and the M.S. and Ph.D. degrees from Stanford University, Stanford, CA, in electrical engineering, in 1989 and 1992, respectively. During his thesis work, he focused on high-speed resonant tunneling and optoelectronic devices.

From 1992 to 1994, he was a Scientist with DOE Ames National Laboratory, Iowa State University, working in the area of PBG materials. He joined the Faculty of Physics, Bilkent University, Ankara, Turkey, in December 1994, where he is currently an Associate Professor. His research with Bilkent involves photonic crystals, silicon micromachining, and high-speed optoelectronics. He has authored or coauthored more than 100 articles in scientific journals, conference proceedings, and books.

Dr. Ozbay is the 1997 recipient of the Adolph Lomb Medal of the Optical Society of America.

Mehmet Bayindir was born in 1975 in Konya, Turkey. He received the B. S. and M. S. degrees in physics from Bilkent University, Ankara, Turkey, in 1995 and 1997, respectively. During his M.S. thesis work, he focused on impurity effects in high-temperature superconductivity, localization in quantum Hall systems, and Bose-Einstein condensation in low-dimensional systems.

He has been working on PBG materials as a Research Assistant in the Department of Physics, Bilkent University, Ankara, Turkey, since 1999. He has authored or coauthored more than 30 articles in scientific journals, conference proceedings, and books.

Mr. Bayindir is one of the 2001 recipients of the New Focus Student Award of Optical Society of America. He has been a member of the American Physical Society since 1995 and a member of Optical Society of America since 1998.

Irfan Bulu was born in 1977 in Malatya, Turkey. He received the B.S. degree in physics from Bilkent University, Ankara, Turkey, in 2001.

He has been working with Prof. Ekmel Ozbay on PBG materials as a Research Assistant at Bilkent University since 2001. He has authored or co-authored five articles in scientific journals, conference proceedings, and books.

Ertugrul Cubukcu was born in 1979 in Kadirli, Turkey. He received the B.S. degree in physics from Bilkent University, Ankara, Turkey, in 2001.

He has been working with Prof. Ekmel Ozbay on PBG materials as a Research Assistant at Bilkent University since 2001. He has authored or coauthored five articles in scientific journals, conference proceedings, and books.

Mr. Cubukcu has been a member of the American Physical Society since 1999 and a member of Optical Society of America since 2000. 